\title{
Warfarin-associated Embryopathy in a 17-week-old Abortus 1
}

\author{
MASON BARR, JR. AND ALPHONSE R. BURDI \\ Teratology Unit, Departments of Pediatrics and Anatomy, University of \\ Michigan, Ann Arbor, Michigan 48109
}

\begin{abstract}
Histological and anthropometric studies were made of the hands and face of a 17-week-old (postmenstrual) human fetus that was exposed to warfarin throughout gestation. The fetus had marked nasal hypoplasia and the facial cartilages contained discrete foci of abnormal cartilage. When compared with agematched control fetuses hand pattern profile analysis showed that the brachydactyly was due to shortening of the terminal phalanges. Histologically chondrogenesis was disordered in the carpals and phalanges, with the most deviant development occurring at the distal ends of the terminal phalanges. In none of the areas of abnormal cartilage was there evidence of hemorrhage or hemosiderin deposition. It is our contention that warfarin is teratogenic in human beings and that its teratogenicity is not due to focal hemorrhage.
\end{abstract}

Several reports have appeared in the literature suggesting an association between maternal ingestion of sodium warfarin and facial and skeletal abnormalities in the offspring (DiSaia, '66; Kerber et al., '68; Tejani, '73; Becker et al., '75; Fourie and Hay, '75; Pettifor and Benson, '75; Shaul et al., '75; Pauli et al., '76; Richman and Lahman, '76.) We wish to describe a 17-weekold aborted fetus that had been exposed to warfarin throughout gestation and to add to the literature our findings of abnormal chondrogenesis.

\section{CASE REPORT}

Mother. The mother was a 26-year-old gravida 2, para 1 who had idiopathic hypertrophic subaortic stenosis and mitral insufficiency (functional class III). For six months before conception and until one week before delivery she took sodium warfarin, $7.5 \mathrm{mg}$ daily, and propanolol, $20 \mathrm{mg}$ three times daily. Because of progressive dyspnea during pregnancy it was decided to terminate the pregnancy. One week before expected delivery the propanolol and warfarin were discontinued and hydrochlorothiazide and KC1 were started. At 17 weeks postmenstrual the fetus was aborted, 23 hours after intraamniotic installation of hypertonic saline. Nine years previously and before the onset of her cardiac disease, the woman, in her first pregnancy, had a normal female infant who has continued to develop normally.

Fetus. The fetus, a female, was macerated, and had a weight of $110 \mathrm{~g}$, crownheel length of $183 \mathrm{~mm}$, crown-rump length of $127 \mathrm{~mm}$, and foot length of $22 \mathrm{~mm}$. These measurements were appropriate for a fetus of $16 \pm 1$ weeks postmenstrual. The face was distinctly abnormal with hypoplasia of the midface, large protuberant eyes covered partially with unfused eyelids, a broad nose (both at the root and alae), excessive bimalar width, widened intercanthal distance, and a relatively large mandible (fig. 1). The fingers appeared to be shorter than normal. Dissection of the fetus after formalin fixation showed no gross visceral malformations. There was extensive autolysis of the brain with partial extrusion of brain substance through a perforation in the posterior fontanelle. The

\footnotetext{
Received March 24, '76. Accepted May 17, '76.

I Supported by NIH Grant DE03443 and by the Beatrice Stanton Medical Research Fund, The University of Michigan Medical School.
} 


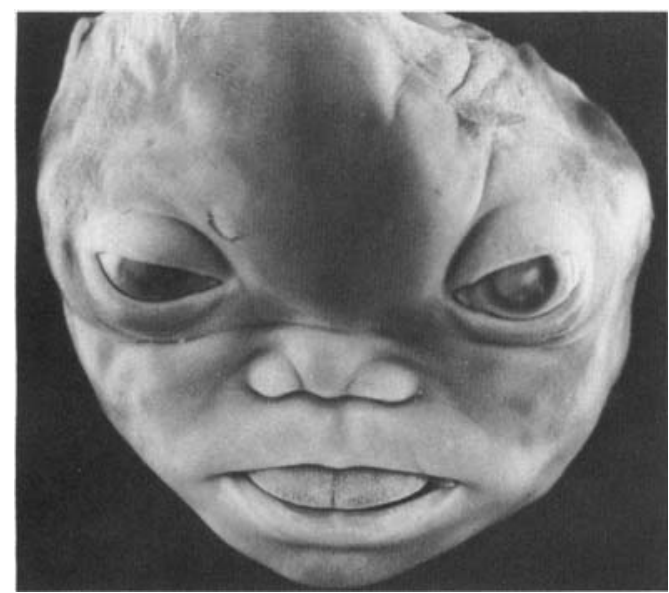

Fig. 1 Frontal view of warfarin-exposed fetus. Note the broadened nose, widened intercanthal distance, large, protuberant eyes, and general hypoplasia of the midface.

placenta (132 g) showed no atypical morphology of either the blood vessels or cord attachment.

\section{FINDINGS}

Recognition of the midfacial hypoplasia prompted a closer study of the fetal skeletal tissues. The face and hand-wrist regions were used for this purpose. Unfortunately, since at the time we were unaware of the possible association of warfarin with chondrodysplasia punctata, the other portions of the macerated body were discarded after dissection of the viscera. Faces and hands of nine morphologically normal fetuses from the Patten Human Embryology Collection (University of Michigan) were used as normal controls. These fetuses were from spontaneous and therapeutic abortions and were closely matched to the warfarin-exposed fetus in age, weight, and crown-rump length.

The fetal tissues were prepared for light microscopy using conventional histological procedures and trichrome connective tissue staining. These preparations were used for (1) a comparison of skeletal tissues in the affected and normal fetuses, and (2) quantitative analyses of finger size, i.e., the hand pattern profile.
Histological examination of the hands indicated that the primary abnormality was in proliferating cartilage. Except for distal phalanx 5 the deviations from normal became more pronounced beyond the metacarpals, into the row of distal phalanges. Cells of the growth cartilage appeared not to have been aligned into regular columns or to have passed through the typical stages of proliferation, maturation, regression, and invasion by capillaries. Instead, irregular masses of chondrocytes were present, producing thick, nodular, and bulky epiphyses.

Distal phalanges 1-4 were most deviant from the control specimens. The most striking peculiarity of each of the four abnormal distal phalanges was the aberrant clump of chondrocytes connecting the shaft with the osteoid tuft (fig. 2A). The base of this clump consisted of immature or proliferative chondrocytes that appeared to align and stream both distally toward the peninsula of cartilage extending into the tuft and proximally toward the shaft of the phalanx (fig. 2B). Distal phalanx 5 more closely resembled the normal picture (fig. $2 \mathrm{C}, \mathrm{D})$.

The disturbance in early skeletogenesis was less pronounced moving toward the carpal rows. The only remarkable feature of the middle phalanges was a marked reduction in circumference of the bone along a line between the proximal epiphyseal area and the bone shaft. This gave the appearance of an attenuated waistline. The only remarkable deviations from normal in the carpal region were small, irregularly placed clumps of cartilage projecting from various carpals. These projections were covered by the perichondrium and gave the carpals irregular shapes.

Hand pattern profiling was used to demonstrate graphically the departure from "normal" of finger size of the warfarinexposed fetus. Anthropometric pattern profiling has been well described and successfully used by anthropologists and pediatric radiologists to show skeletal abnormalities (Garn et al., '72; Poznanski et al., '72). In this technique the dimensions of 

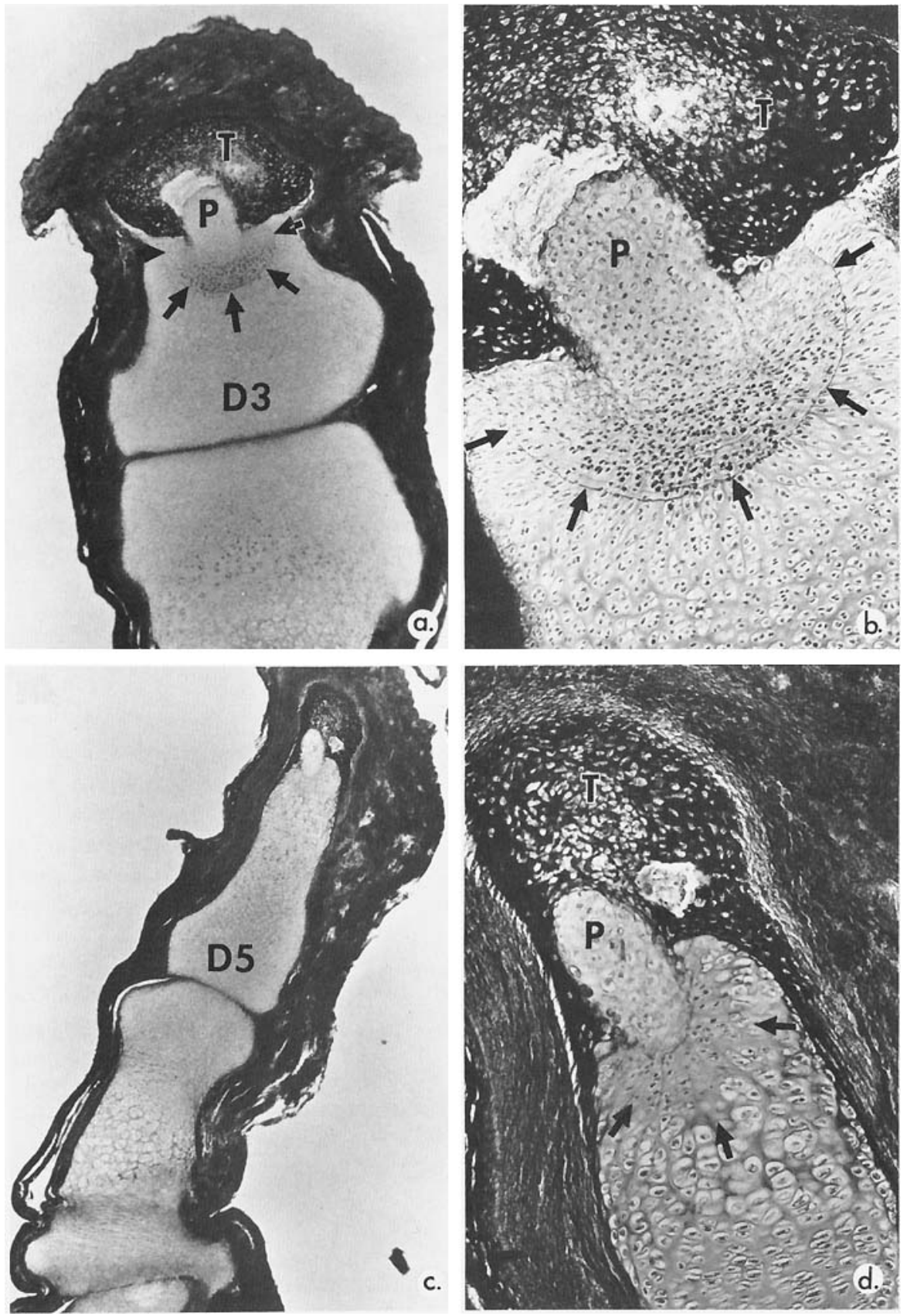

Fig. 2 a. Distal phalanx 3 . Note the shortened length, nodular tuft (T), and arc of proliferating, immature chondrocytes (arrows) at the base of the peninsular cartilage (P). $\times 5$.

b. Distal phalanx 3. This enlargement of the arc proliferation field (arrows) shows the dividing chondrocytes with columns of mature chondrocytes radiating both distoproximally and proximodistally. The latter direction is the typical arrangement. Also note the peninsula $(\mathrm{P})$ of mature cartilage interlocked with the tuft $(\mathrm{T}) . \times 50$.

c. Distal phalanx 5. The fifth digit appears less affected than distal phalanges 1-4 and is not foreshortened. $\times 5$.

d Distal phalanx 5 . This enlargement of the distal region shows the presence of proliferating, immature chondrocytes (arrows) at the base of the peninsular cartilage (P). Both of these represent a microform of conditions seen in distal phalanges $1-4 . \times 50$. 
skeletal structures are expressed relative to the mean and standard deviation of dimensions for a reference population. The fetal hand pattern profile is based on maximum bone lengths of each long bone of the hands, using microprojection (controlled for distortion) of serial hand sections and measurement with vernier calipers.

The maximum length of the long bones of the hands of the nine normal agematched abortuses was measured and the mean lengths and standard deviations were calculated. The pattern profile of the affected fetus was developed by determining the deviation from normal in standard deviation units and plotting the resulting $z$ scores on a graph (fig. 3). The metacarpals and the proximal and middle phalanges of the warfarin-exposed fetus were somewhat short but were within the $95 \%$ confidence limits of the mean. Distal phalanges 1-4 were below the 2.5 centile and were therefore considered abnormally short. Distal phalanx 5 was not significantly shorter than normal.

In the facial cartilages several foci of disordered chondrogenesis were found (fig. 4). These foci consisted of well-circumscribed areas in which the matrix was more intensely stained than the normal matrix.
There was also a separation of the central borders of these foci from the surrounding matrix. In those areas of cartilage in which rays of chondrocytes were evident the rays extended across the borders and into the abnormal areas. No deviations from normal cellular architecture were evident on light microscopy.

The possibility that the abnormal foci were artifacts or due to autolysis was considered, but since we have not found them in other specimens prepared in the same way or in specimens with equal or greater degrees of autolysis we think the changes we observed are pathological. The lack of hemosiderin deposition in or around the abnormal areas indicates that focal hemorrhage was not responsible for the disordered chondrogenesis.

\section{DISCUSSION}

Several reports have implicated sodium warfarin as a human teratogen (DiSaia, '66; Kerber et al, '68; Tejani, '73; Becker et al., 75; Fourie and Hay, '75; Pettifor and Benson, '75; Shaul et al., '75; Pauli et al., '76; Richman and Lahman, '76.) All the cases had hypoplasia of the nasal bones, most had some stippling of the epiphyses on roentgenographic examination, and several

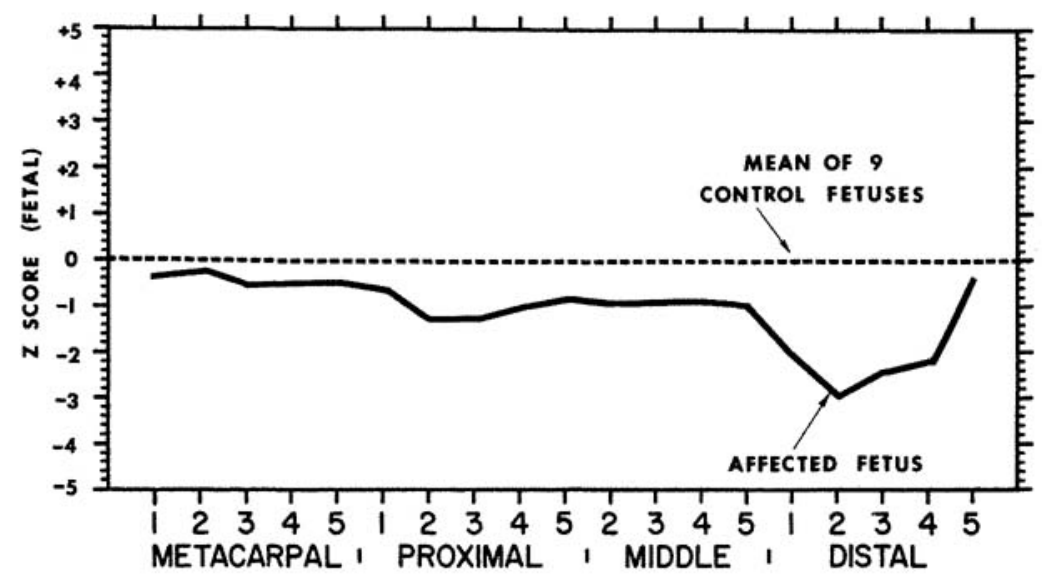

Fig. 3 Hand pattern profile of the warfarin-exposed fetus. $z$ values (standard deviations) are on the ordinate and individual bones are on the abcissa. The dashed line at $z=0$ is the pattern profile derived for the control sample of nine fetuses matched for crown-rump length with the affected fetus. The solid line is the pattern profile of the warfarin-exposed fetus. The small proximals, middles, and distals, with the exception of distal phalanx 5 , departed significantly from the expected value. 

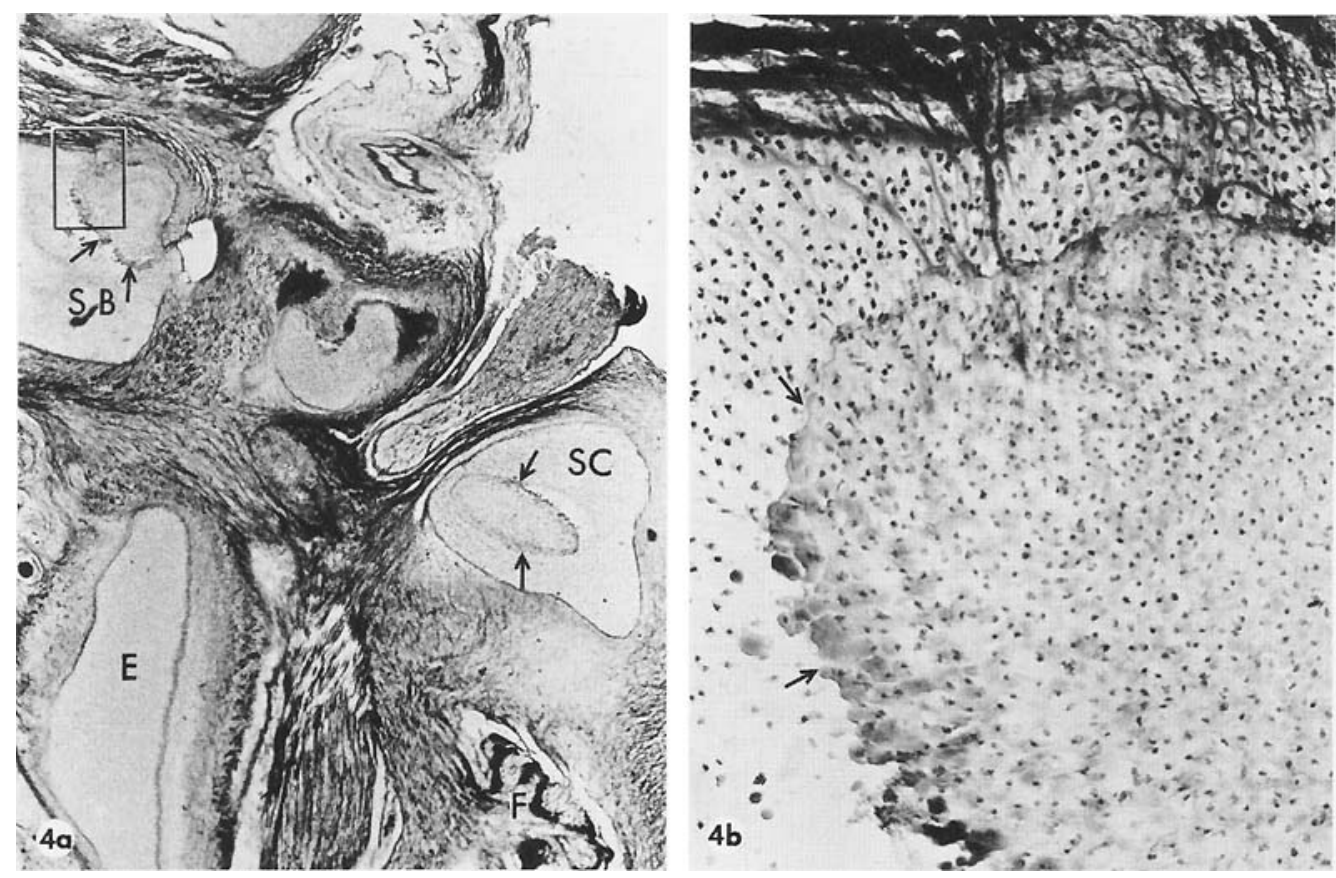

Fig, 4 a. Cranial base region. Note the presence of atypical cartilage proliferations in different regions of the sphenoid (arrows). In this photo both the sphenoid crest (SC) and the body of the sphenoid (SB) show this atypical cartilage condition. Shown also are the ossified frontal bone (F) and ethmoid cartilage $(E)$. The ethmoid also showed indications of this atypical cartilage condition. $\times 2$.

b. Body of sphenoid. Enlargement of region enclosed in box in figure 4a. Note the superimposition of a darker staining cartilage field (arrows) over a typical, lighter staining cartilage field. $\times 20$.

had brachydactyly. Although pregnancy wastage is high among women on anticoagulants the complex of nasal hypoplasia, stippled epiphyses, and brachydactyly has not, with one exception, been reported in the offspring of women on other coumarin anticoagulants (phenindione, dicumerol, and acencumarin) (Tejani, '73). The one exception was case 1 of Pettifor and Benson ('75) where the mother took phenindione and the description of that infant was typical for infants exposed to warfarin. It is of interest to note that the mother also took diphenylhydantoin and primidone, drugs that have been associated with digital hypoplasia and facial abnormalities (Loughnan et al., '73; Barr et al., '74; Hanson and Smith, '75.)

It has been suggested that the teratogenic effect of warfarin may be related to focal bleeding in fetal or embryonic car- tilage, which would lead to abnormal cartilage growth and scarring (Becker et al., 75). The scarred areas would presumably calcify, producing stippling of the epiphyses. Our case demonstrates a basic disorder in chondrogenesis that suggests to us that the stippling is not due to focal hemorrhage but to disorganization of chondrogenesis producing islands of cartilage that calcify in advance of the surrounding cartilage. Also supporting this concept is the histological examination of case 2 of Becker et al. ('75); they found well-delineated foci of atypical cartilage that consisted of areas of proliferating small chondrocytes with calcification of the matrix and areas of acellular myxomatous material with numerous thin-walled vascular channels. Because of the apparent risk to the fetus from exposure to warfarin we concur with the advice of Tejani ('73) that 
if at all possible the use of warfarin be avoided during the first trimester of pregnancy. Whether or not use of warfarin during the second trimester is safe is uncertain. We are aware of reports concerning four infants born with brain abnormalities to mothers who were started on coumarin anticoagulants after the first trimester. (Quenneville et al., '59; Warkany and Bofinger, '75; Sherman and Hall, '76.) It is also uncertain at this time whether or not other anticoagulants of the coumarin group have a lower risk of teratogenesis or if simultaneous use of other medications potentiates the teratogenicity of warfarin.

\section{LITERATURE CITED}

Barr, M., Jr., A. K. Poznanski and R. D. Schmickel 1974 Digital hypoplasia and anticonvulsants during gestation: a teratogenic syndrome? J. Pediat., 84 : 254-256.

Becker, M. H., N. G. Genieser, M. Finegold, D. Miranda and T. Spackman 1975 Chondrodysplasia punctata: is maternal warfarin therapy a factor? Am. J. Dis. Child., 129: 356-359.

DiSaia, P. J. 1966 Pregnancy and delivery of a patient with a Starr-Edwards mitral valve prosthesis. Obst. Gyn., 28: 469-472.

Fourie, D. T., and I. T. Hay 1975 Warfarin as a possible teratogen. S. Afr. Med. J., 49: 2081-2083.

Garn, S. M., K. P. Hertzog, A. K. Poznanski and I. M. Nagy 1972 Metacarpophalangeal length in the evaluation of skeletal malformation. Radiology, 105: $375-381$.
Hanson, J. W., and D. W. Smith 1975 The fetal hydantoin syndrome. J. Pediat., 87: 285-290.

Kerber, I. J, O. S. Warr and C. J. Richardson 1968 Pregnancy in a patient with a prosthetic mitral valve. J. Am. Med. Ass., 203: 223-225.

Loughnan, P. M., H. Gold and J. C. Vance 1973 Phenytoin teratogenicity in man. Lancet, 1; 70-72.

Pauli, R. M., J. D. Madden, K. J. Kranzler, W. Culpepper and R. Port 1976 Warfarin therapy initiated during pregnancy and phenotypic chondrodysplasia punctata. J. Pediat., 88: 506-508.

Pettifor, J. M., and R. Benson 1975 Congenital malformations associated with the administration of oral anticoagulants during pregnancy. J. Pediat., 86 : 459-462.

Poznanski, A. K., S. M. Garn, J. M. Nagy and J. C. Gall, Jr. 1972 Metacarpophalangeal pattern profiles in the evaluation of skeletal malformations. Radiology, 104: 1-11.

Quenneville, G., B. Barton, E. McDevitt and I. S. Wright 1959 The use of anticoagulants for thrombophlebitis during pregnancy. Am. J. Obst. Gyn., 77: 1135-1149.

Richman, E. M., and J. E. Lahman 1976 Fetal anomalies associated with warfarin therapy initiated shortly prior to conception. J. Pediat., 88: 509-510.

Shaul, W. L., H. Emery and J. G. Hall 1975 Chondrodysplasia punctata and maternal warfarin use during pregnancy. Am. J. Dis. Child., 129: 360362 .

Sherman, S., and B. D. Hall 1976 Warfarin and fetal abnormality. Lancet, $1: 692$.

Tejani, N. 1973 Anticoagulant therapy with cardiac valve prosthesis during pregnancy. Obst. Gyn., 42: 785-793.

Warkany, J., and M. Bofinger 1975 Le rôle de la coumadine dans les malformations congenitales. Med. Hyg., 33: 1454-1457. 